\title{
High-Energy Neutrino Signatures of Newborn Pulsars In the Local Universe
}

\author{
Ke Fang \\ Department of Astronomy \& Astrophysics, Kavli Institute for Cosmological Physics, The \\ University of Chicago, Chicago, Illinois 60637, USA.
}

\begin{abstract}
Charged particles can be accelerated to higher than PeV energies in the electromagnetic wind of a fast-spinning newborn pulsar to produce high-energy neutrinos, through hadronuclear interactions in the supernova remnant. Here we explore the detectability and observational signatures of these high-energy neutrinos. We show that their spectral index varies approximately from 1.5 to 2 , depending on the relevant pulsar properties and observation time. We also apply the scenario to existing young pulsars in the local universe and find the corresponding neutrino flux well below current detection limits. Finally, we estimate the birth rate of fast-spinning pulsars in the local Universe that can be observed by the IceCube observatory to be 0.07 per year, with an upper limit of 0.29 per year.
\end{abstract}




\section{Contents}

1 Introduction 1

2 Neutrino production mechanism $\quad 2$

3 Numerical Setup $\quad 4$

4 Time-integrated Neutrino Spectrum $\quad 7$

5 Time-Dependent Neutrino Emissions $\quad 9$

5.1 Flux level and Detectability 9

5.2 Existing nearby pulsars 11

6 Birth rate of detectable pulsars $\quad 11$

6.1 Upper limit 13

6.2 Pulsars in nearby clusters 14

6.3 Pulsars in the Local Group $\quad 15$

7 Conclusions and Discussion $\quad 15$

\section{Introduction}

Fast-spinning pulsars are known to release their rotational energy via electromagnetic radiation [1]. Charged particles, including heavy ions [2, 3], can be accelerated by energy conversion of the wind Poynting flux into kinetic energy in pulsar winds [4-6]. When cosmic rays escape from the acceleration site, they interact with the surrounding supernova ejecta [7]. High-energy neutrinos are then produced during hadronuclear interactions. Such high-energy neutrinos are among the first multimessenger signals released by the star, and in addition, they experience no delay in galactic and intergalactic magnetic fields. Hence a measurement of the high-energy neutrino signal from a future event can provide a unique way to identify the birth of a newborn pulsar and the start of high-energy acceleration in that system.

The IceCube Observatory recently reported the detection of a diffusive flux of highenergy astrophysical neutrinos $[8,9]$. These 37 events have deposited energies between $\sim$ $50 \mathrm{TeV}$ and $2 \mathrm{PeV}$, and are consistent with an isotropic $E^{-2}$ spectrum. Meanwhile, searches for point-like neutrino sources, applying four years of IceCube data, still find the event data to be compatible with the background signal $[10,11]$. No point sources of high-energy neutrinos have been identified thus far.

We showed that a diffusive $\mathrm{EeV}\left(10^{18} \mathrm{eV}\right)$ neutrino flux should be effectively produced if extragalactic newborn pulsars are the emitters of ultrahigh energy cosmic rays [12]. In this work we consider, more generally, the $\mathrm{TeV}\left(10^{12} \mathrm{eV}\right)$ to EeV high-energy neutrino emissions from individual fast-spinning pulsars in the local universe, focusing on their light curve, energy spectrum, and flux detectability, especially in light of recent IceCube measurements. In particular, we extend the previous work of [13] to a study with complete pulsar population including magnetars and Crab-like pulsars, and update those results based on a full Monte Carlo simulation. We also apply the scenario to existing nearby pulsars and find their emission 
levels to be consistent with the non-detection. Based on the discovery sensitivities of IceCube, we provide a prediction of the detectability and a template of emission profiles for high-energy neutrinos from a future nearby newborn pulsar.

Section 2 describes the neutrino production mechanism. Following a description of the numerical setup of this work in Section 3, the results of time-integrated neutrino spectrum is presented in Section 4, focusing on the effects from secondary interactions and the dependence on pulsar properties. Section 5 presents the time evolution of the neutrino emissions, and provides a template for future detections to identify local pulsar sources. We also apply the scenario to recent nearby pulsar systems and test the agreement to the non-detections of point sources. In Section 6 we investigate the birth rate of future pulsars that could be detected by the IceCube Observatory. We draw conclusions and discuss the implications for future neutrino observations in Section 7.

\section{Neutrino production mechanism}

The idea that cosmic rays could be accelerated by rotating neutron stars dates back to $[1]$. $[5,6]$ further developed the scheme of the production of ultra-high energy cosmic ray in the electric field associated to the rotating magnetic dipole, although uncertainties still exist especially regarding the seeding of heavy ions $[2,3]$ and the acceleration sites $[6,7]$. Here we review the cosmic ray production process with energy dissipation by electron pairs additionally taken into account, and calculate the corresponding neutrino emissions.

Considering a newborn pulsar spinning down and releasing rotational energy by electromagnetic radiation, its characteristic spin-down time reads $\tau_{\mathrm{EM}}=\left(1 / 2 I \Omega_{i}^{2}\right) / \dot{E}_{\mathrm{rot}}=$ $1 B_{13}^{-2} P_{i,-3}^{2}$ yr, where $\dot{E}_{\text {rot }}=4 \mu^{2} \Omega_{i}^{4} / 9 c^{3}$ is the dipole radiation rate for a pulsar with inertia $I \approx 10^{45} \mathrm{~g} \mathrm{~cm}^{2}$, surface magnetic field $B=10^{13} B_{13} \mathrm{G}$ and initial spin period $P_{i}=1 P_{i,-3} \mathrm{~ms}$. Please note that in the following text, two corresponding parameters - magnetic moment $\mu=B R^{3} / 2 \approx 10^{31} B_{13} \mathrm{erg} / \mathrm{G}$ and initial rotation speed $\Omega_{i}=2 \pi / P_{i}=10^{3.8} P_{i,-3}^{-1} \mathrm{~s}^{-1}$, might be used as alternatives to $B$ and $P$. We assume that the electromagnetic energy is efficiently converted into the kinetic energy of charged particles including ions and electrons, with a rest frame injection rate [14]

$$
\dot{N} m c^{2}=\frac{\dot{N}_{\mathrm{GJ}}}{Z} m_{i} c^{2}+2 \kappa \dot{N}_{\mathrm{GJ}} m_{e} c^{2}
$$

Here $\dot{N}_{\mathrm{GJ}}=\sqrt{\dot{E}_{\mathrm{rot}}} \mathrm{c} / e=\mu \Omega^{2} / e c$ is the Goldreich-Julian rate [15], that is the number of elementary charges per unit volume that enter the parallel electric fields in the magnetosphere, $m_{i}=A m_{p}$ and $Z$ are the mass and the charge of the ion, and $\kappa$ is the pair-production multiplicity (see for e.g. [16]). Let $y_{i} \equiv 2 Z \kappa m_{e} / m_{i}$, which can be interpreted as the ratio of the kinetic energy that goes into electrons to that goes into ions. In crab-like pulsars $\kappa \sim 10^{4}$ [17], then $y_{i} \sim 10$.

If a fraction $\eta$ of the electromagnetic luminosity turns into the kinetic luminosity, an ion injected at system time $t$ would obtain energy of

$$
E_{\mathrm{CR}}(t)=\frac{\eta \dot{E}_{\mathrm{rot}}(t)}{\dot{N} m c^{2}} m_{i} c^{2}=E_{\mathrm{CR}, \max }\left(1+t / \tau_{\mathrm{EM}}\right)^{-1}
$$


where

$$
\begin{aligned}
E_{\mathrm{CR}, \max } & =\eta Z e \frac{\Omega_{i}^{2} \mu}{c^{2}} \frac{1}{1+y_{i}} \\
& \approx 1.7 \times 10^{18} B_{13} P_{i,-3}^{-2} \eta_{-0.5} A \kappa_{4}^{-1} \mathrm{eV} \quad \mathrm{y}_{\mathrm{i}} \gg 1
\end{aligned}
$$

is the maximum energy the ion can gain from the induced electric potential $[5,6]$. Note that we have taken an acceleration efficiency $\eta=0.3 \eta_{-0.5}$ as suggested by $[17,18]$.

Notice that the conduction currents in the pulsar wind have a charge density proportional to the particle energy $\dot{N}_{\mathrm{GJ}} \propto E_{\mathrm{CR}}$. As a consequence, the prompt particle injection rate is a constant over time:

$$
\frac{d N_{\mathrm{CR}}}{d E d t}=\frac{c}{(Z e)^{2} \eta}\left(1+y_{i}\right)
$$

Integrating it over the corresponding acceleration period $\Delta t(E)=\left(E_{\mathrm{rot}} \eta /\left(1+y_{i}\right)\right) / \dot{E}_{\mathrm{rot}}$, one gets the time-integrated energy spectrum

$$
\frac{d N_{\mathrm{CR}}}{d E}=\frac{9}{8} \frac{c^{2} I}{Z e \mu} \frac{1}{E_{\mathrm{CR}}}
$$

Notice that in the above calculations we ignored the gravitational wave losses, which would only become significant for neutron stars with surface magnetic field $B \gg 10^{15} \mathrm{G}[6,19]$.

After accelerated in the pulsar winds, particles would cross the supernova ejecta surrounding the star at $R_{\mathrm{ej}}(t)=3 \times 10^{16} \beta_{\mathrm{ej},-1.5} t_{\mathrm{yr}} \mathrm{cm}$ to escape from the source. The ejecta can be modeled as a shell spherically expanding with speed $\beta=\left(\left(1 / 2 I \Omega_{i}^{2}+E_{\mathrm{ej}}\right) / M_{\mathrm{ej}} c^{2}\right)^{1 / 2}=$ $0.03 \beta_{-1.5}$ and column density $\Sigma_{\mathrm{SN}}(t)=5.3 M_{\mathrm{ej}, 1} \beta_{-1.5}^{-2} t_{\mathrm{yr}}^{-2} \mathrm{~g} \mathrm{~cm}^{-2}[7]$, where $M_{\mathrm{ej}, 1}=10 M_{\mathrm{ej}} / M_{\odot}$ is the ejecta mass and $E_{\text {ej }}$ is the ejecta energy that includes both star's rotational and explosion energy.

In an early environment, cosmic rays would interact with the dense ejecta through hadronuclear interactions, $N+p \rightarrow N^{\prime}+\pi+$ others. The effective optical depth reads $\tau=n_{b} \sigma \kappa R_{\mathrm{ej}}$. For protons, $\tau_{\mathrm{pp}}=0.16 M_{\mathrm{ej}, 1} \beta_{-1.5}^{-2} t_{\mathrm{yr}}^{-2}$, with $\sigma_{\mathrm{pp}} \sim 10^{-25} \mathrm{~cm}^{2}, \kappa \sim 0.5$ being the cross section and elasticity of proton-proton interaction at EeV. For nuclei, since $\sigma_{N p} \propto A^{2 / 3} \sigma_{\mathrm{pp}}$ and $\kappa \propto 0.7 / A$, the effective optical depth for nuclei $\left(\tau_{\mathrm{Np}}\right)$ is reduced by $\sim A^{1 / 3}$. The system becomes optically thin after $\tau_{\text {thin }} \equiv t\left(\tau_{\mathrm{pp}}=1\right)=0.4 M_{\mathrm{ej}, 1}^{1 / 2} \beta_{-1.5}^{-1} \mathrm{yr}$. The pion production efficiency is therefore

$$
f_{\pi}=\min \left(\tau_{\mathrm{Np}}, 1\right)
$$

Charged pions produced in the hardonuclear interactions have a relatively short life time $\tau_{\pi}=2.6 \times 10^{-8} \mathrm{~s}$ in rest frame. However, at early stages the ejecta would so dense that the pions interact with the baryons before they decay. The $\pi p$ interaction time reads $t_{\pi p}=\left(n_{b} \sigma_{\pi p} \kappa_{\pi p} c\right)^{-1}$, with cross section $\sigma_{\pi p}=5 \times 10^{-26} \mathrm{~cm}^{2}$ and elasticity $\kappa_{\pi p} \sim 0.5$ [20]. The probability that a $\pi p$ interaction happens before the pion decays introduces a suppression factor,

$$
f_{\text {sup }}=1-e^{-t_{\pi p} / \gamma_{\pi} \tau_{\pi}}
$$

which can be approximated by $f_{\text {sup }} \approx t_{\pi p} / \gamma_{\pi} \tau_{\pi}$ when $t_{\pi p} \ll \gamma_{\pi} \tau_{\pi}$. Notice that the hadronic cooling of muons is negligible compared to that of pions since $\sigma_{\mu p} \approx 2 \times 10^{-28} \ll \sigma_{\pi p}[20]$. Finally charged pions would decay into neutrinos via $\pi^{ \pm} \rightarrow e^{ \pm}+\nu_{e}\left(\bar{\nu}_{e}\right)+\bar{\nu}_{\mu}+\nu_{\mu}$ after ejecta gets thin. After taking into account that the pion carries about $20 \%$ of the proton's energy 
in a pp interaction, and that each of the neutrino inherits a quarter of the charged pions energy, a characteristic energy of the neutrino products is $E_{\nu} \approx 0.05 E_{p}$. Taking along the suppression factor, the neutrino flux produced by hadronuclear interactions in the ejecta can be calculated as

$$
E_{\nu}^{2} \Phi_{\nu}=\frac{3}{8} E_{\mathrm{CR}}^{2} \Phi_{\mathrm{CR}} f_{\pi} f_{\text {sup }}
$$

The flux should reach a peak before ejecta getting thin at $\tau_{\text {thin }}$, but after the suppression from $\pi p$ interaction turning relatively weak when $t_{\pi p}=\gamma_{\pi} \tau_{\pi}$. Assuming $E_{\pi, \max }=$ $0.2 E_{\mathrm{CR}, \max }$, the latter condition is equivalent to

$$
t_{\text {peak }}^{3}\left(1+\frac{t_{\text {peak }}}{\tau_{\mathrm{EM}}}\right)=\frac{E_{\pi, \max }}{m_{\pi} c^{2}} \tau_{\pi} \frac{M_{\mathrm{ej}} \sigma_{\pi p} c}{\frac{4}{3} \pi m_{b}(\beta c)^{3}}
$$

Below we estimate the peak time and the peak energy with two representative cases: I) a fast-spinning magnetar with $P_{i}=0.6 P_{-3.2} \mathrm{~ms}$ and $B=10^{15} B_{15} \mathrm{G}$, located at a distance $D=5 \mathrm{Mpc}$. We will call this case magnetar; II) a crab-like pulsar with $B=10^{13} B_{13} \mathrm{G}$ and $P_{i}=20 P_{-1.7} \mathrm{~ms}$, located at $D=10 \mathrm{kpc}$. We denote this case as crab. In the magnetar case, $t_{\text {peak }} \gg \tau_{\mathrm{EM}}=3 \times 10^{3} \mathrm{~s}$, equation 2.9 raises

$$
\begin{aligned}
t_{\text {peak }} & =2.4 \times 10^{5} B_{15}^{-1 / 4} A^{1 / 4} \kappa_{4}^{-1 / 4} \eta_{-0.5}^{1 / 4} M_{\mathrm{ej}, 1}^{1 / 4} P_{i,-3.2}^{3 / 4} \mathrm{~s} \\
E_{\nu, \text { peak }} & =1.1 \times 10^{17} B_{15}^{-3 / 4} \eta_{-0.5}^{3 / 4} A^{3 / 4} \kappa_{4}^{-3 / 4} M_{\mathrm{ej}, 1}^{-1 / 4} P_{i,-3.2}^{-3 / 4} \mathrm{eV}
\end{aligned}
$$

In contrast, in the crab case $t_{\text {peak }} \ll \tau_{\mathrm{EM}}=1.2 \times 10^{10} \mathrm{~s}$ instead, and

$$
\begin{aligned}
t_{\text {peak }} & =2.1 \times 10^{5} B_{13}^{1 / 3} P_{i,-1.7}^{-2 / 3} A^{1 / 3} \kappa_{4}^{-1 / 3} \eta_{-0.5}^{1 / 3} M_{\mathrm{ej}, 1}^{1 / 3} \mathrm{~s} \\
E_{\nu, \text { peak }} & =2.1 \times 10^{14} B_{13} P_{i,-1.7}^{-2} \eta_{-0.5} A \kappa_{4}^{-1} \mathrm{eV}
\end{aligned}
$$

\section{Numerical Setup}

The interactions between high-energy nuclei and ejecta baryons were simulated by Monte Carlo as we did in [7]. In particular, the $\mathrm{Np}$ and pp interactions were calculated based on the hadronic interaction model EPOS [21] and the fragmentation model as implemented in the air shower simulation code CONEX [22]. For this work, in order to take into account the suppression in neutrino production, we further adapted the code with pion-proton interactions calculated using EPOS.

In Figure 1 we show a histogram of neutrino and electron products of $\pi p$ interaction between a $1 \mathrm{EeV} \pi^{+}$and a rest proton in the lab frame. The flux ratio between electron, electron neutrino and muon neutrino before mixing is consistent with $\Phi_{e}: \Phi_{\nu_{e}}: \Phi_{\nu_{\mu}}=1: 1: 2$ as expected from the decay of charged pions. Note that this result is obtained by allowing all intermediate pions and muons to fully decay to leptons. This might not be true in an intense interaction environment. As Equation 2.7 shows, a suppression would happen as pions are tend to interact rather than decay when the ejecta is still dense. Remember that the elasticity of $\pi p$ interaction at EeVs is about 0.5 [13], so roughly half of the pion's energy is passed to additional interactions that produce extra lower energy neutrinos.

In the simulations, we modeled pulsars with selected initial rotation speed $\Omega_{i}$ and magnetic moment $\mu$. To calculate time-integrated neutrino spectrum, we injected cosmic rays following a power-law spectrum as in Equation 2.5, with minimum energy $E_{\min }=10^{12} \mathrm{eV}$ and 


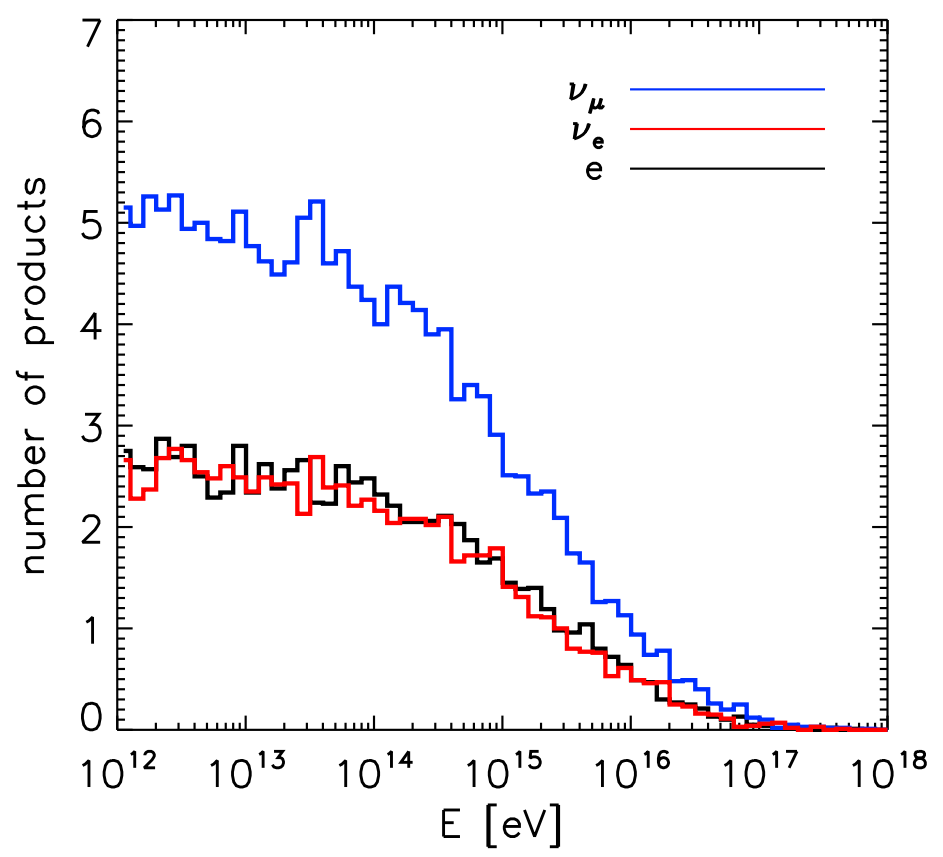

Figure 1. Decay products of $\nu_{e}, \nu_{\mu}$ and $e$ from a $\pi p$ interaction, allowing all pions to decay without further interactions. The injected pion had energy of $1 \mathrm{EeV}$ and the proton was assumed to be at rest in lab frame. The histogram is an average of 100 realizations using the hadronic interaction model EPOS [21].

Table 1. Summary of input parameters

\begin{tabular}{ccccccccc}
\hline \hline Model & $\begin{array}{c}P_{i} \\
(\mathrm{~ms})\end{array}$ & $\begin{array}{c}\mathrm{B} \\
(\text { Gauss })\end{array}$ & $\begin{array}{c}M_{\mathrm{ej}} \\
\left(M_{\odot}\right)\end{array}$ & $\begin{array}{c}E_{\text {exp }} \\
(\mathrm{erg})\end{array}$ & $\eta$ & $\kappa$ & $\begin{array}{c}\mathrm{I} \\
\left(\mathrm{g} \mathrm{cm}^{-2}\right)\end{array}$ & $\begin{array}{c}\text { Distance } \\
(\mathrm{kpc})\end{array}$ \\
\hline Magnetar & 0.6 & $10^{15}$ & 10 & $10^{51}$ & 0.3 & $10^{4}$ & $10^{45}$ & 5000 \\
Crab & 20 & $10^{13}$ & 10 & $10^{51}$ & 0.3 & $10^{4}$ & $10^{45}$ & 10 \\
\hline
\end{tabular}

maximum energy calculated by Equation 2.3. To calculate time-dependent neutrino emission at chosen time $t$, we injected cosmic rays with single energy decided by Equation 2.2 and flux normalized with Equation 2.4. The injected cosmic rays were then propagated through a supernova envelope of total ejected mass $10 M_{\odot}$ expanding at $v_{\mathrm{ej}}=\left(2 E_{\mathrm{ej}} / M_{\mathrm{ej}}\right)^{1 / 2}$. The ejecta energy includes the pulsar's rotational energy and the supernova explosion energy, $E_{\text {ej }}=I \Omega_{i}^{2} / 2+E_{\text {exp }}$. Each time when a pion was produced, the suppression factor in Equation 2.7 was calculated to decide if it would decay or interact, by comparing with a random number drawn from a uniform distribution between 0 and 1 . All the products from primary and higher order interactions, which we will call secondary particles in general, were tracked down to $E_{\min }$. As we will see in Section 4, these secondary particles play a key role in neutrino production. The input parameters used for the magnetar and crab cases are summarized in Table 1, and that for the existing nearby pulsars are listed in Table 2. 

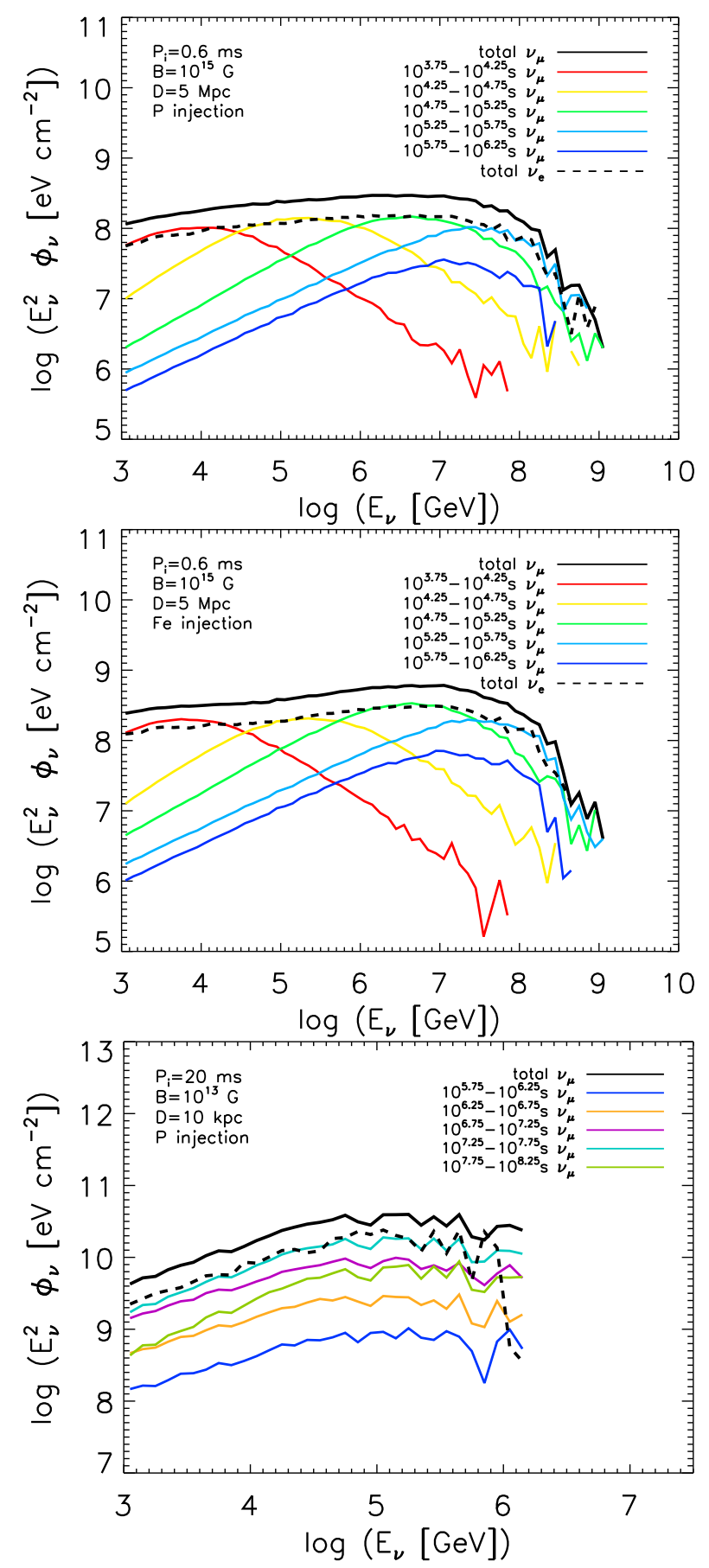

Figure 2. Time-integrated spectrum of muon neutrinos (solid) and electron neutrinos (dash) from newborn pulsars in the local universe. Top and middle: magnetar case with injected cosmic rays being pure proton (top) and iron nuclei (middle) - the source is a fast-spinning magnetar with $P_{i}=0.6 \mathrm{~ms}$ and $B=10^{15} \mathrm{G}$ located at $D=5 \mathrm{Mpc}$. Bottom: crab case with proton injection - the source is a crab-like pulsar with $P_{i}=20 \mathrm{~ms}$ and $B=10^{13} \mathrm{G}$ located at $D=10 \mathrm{kpc}$. The distances of the sources were artificially chosen to fit the plotting scale. The neutrino contributions from different time intervals are listed as in the legend box. 

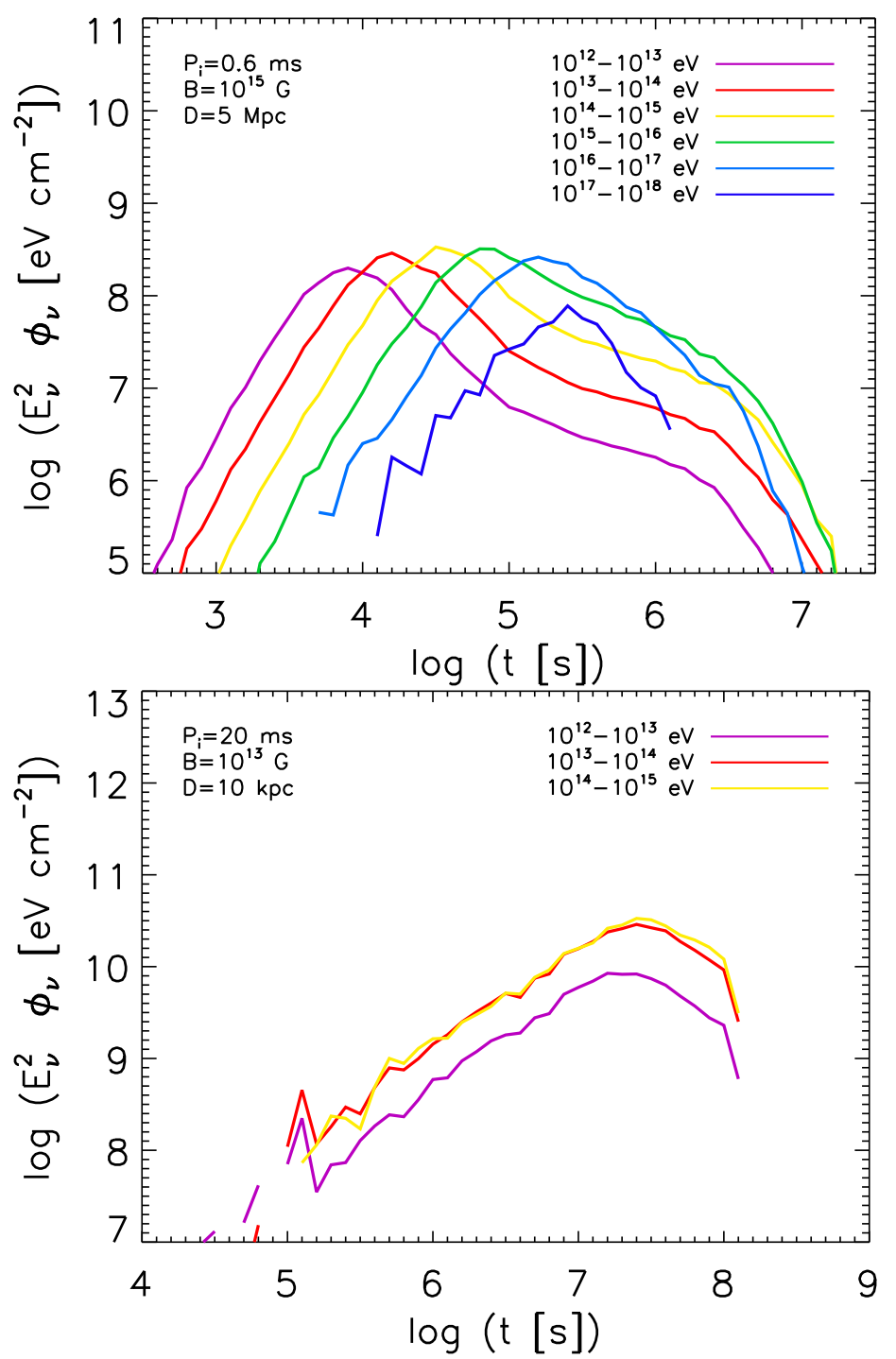

Figure 3. Neutrino light curves of the magnetar case (top) and the crab case (bottom). The input parameters for the two cases are listed in Table 1. Pure proton injection has been assumed. Note that the color codes are different from Fig. 2, and are indicating different neutrino energies as listed in the legend box.

\section{Time-integrated Neutrino Spectrum}

The spin-down time of a pulsar ranges from minutes to thousands of years depending on the star's initial properties. The duration of neutrino emissions could last for the entire spin-down time in principle, but the majority of the neutrinos are produced before the environment gets too thin for interactions. In this section we assume that the observation could last for tens of years to study the neutrino spectrum accumulated over time. We divide the detection period into logarithmic time bins then study the evolution of the spectrum within each interval.

The top panel in Fig 2 shows the expected neutrino spectrum from a newborn magnetar located at $5 \mathrm{Mpc}$ with $P_{i}=0.6 \mathrm{~ms}$ and $B=10^{15} \mathrm{G}$ with pure proton injections. This is a highly magnetized and extremely fast-rotating neutron star that has a rare birth rate. 
In particular, we note that $P_{i}=0.6 \mathrm{~ms}$ roughly reaches the minimum spin period allowed for neutron stars $[7,23]$. However, the short spin-down time $\tau_{\mathrm{EM}} \approx 1000 \mathrm{~s}$ of the magnetar makes it a good representative of pulsars that release energy quickly and force cosmic rays to undergo severe interaction processes. We divide the observation time from $10^{4} \mathrm{~s}$ to $10^{6} \mathrm{~s}$ into 5 logarithmic bins, indicated by corresponding colors as in the legend. In the first two bins centered at $10^{4} \mathrm{~s}$ and $10^{4.5} \mathrm{~s}$, the ejecta is very dense with $\tau_{\pi p} \gg 1$, so the neutrino production is heavily suppressed, as pion products continue to suffer higher order $\pi p$ interactions. The suppression continues till $t_{\pi p} \approx \gamma_{\pi} \tau_{\pi}$, corresponding to a peak at $E_{\nu \text {,sup }}(t)=1.6 \times 10^{13} t_{4}^{3} \beta_{-1}^{3} M_{\mathrm{ej}, 1}^{-1} \mathrm{eV}$. Later at $10^{5} \mathrm{~s}$ and $10^{5.5} \mathrm{~s}$, cosmic rays are accelerated to lower energy, so neutrinos peaked at $\min \left(E_{\nu}(t), E_{\nu, \text { sup }}(t)\right)$. When coming to the last bin centered at $10^{6} \mathrm{~s} \sim \tau_{\text {thin }}$, the flux suffers from both decrease factors where the flux was truncated by $f_{\pi}<1$ and $E_{\nu}$ drops as a result of the spin-down. Unlike the injection spectrum of $E^{-1}$, the overall neutrino spectrum follows $\sim E^{-2}$, because in such a fast-spinning magnetar, most cosmic rays were injected when the environment was still opaque, $\tau_{\mathrm{EM}} \ll \tau_{\text {thin }}$. Thus, the spectrum was heavily boosted by secondary products. Intuitively, if a pion that could have decayed into one primary neutrino with $E_{\nu \text {,prim }}$ eventually fully interacted to $\sim E_{\nu, \text { prim }} / E_{\nu, \text { sec }}$ secondary neutrinos, each with energy $E_{\nu, \text { sec }}$, the secondary flux would satisfy $\left(E_{\nu} d N / d E\right)_{\mathrm{sec}}=\left(E_{\nu} d N / d E\right)_{\text {prim }} \times\left(E_{\nu, \text { prim }} / E_{\nu, \text { sec }}\right) \propto E_{\nu, \text { sec }}^{-1}$. Note that our result is quite different from Fig. 1 of [13] below $\sim 10 \mathrm{PeV}$, as the neutrino spectrum significantly changes when the secondary $\mathrm{Np}$ and $\pi p$ interactions are taken into account.

The spectrum and flux of neutrinos of hadronic origin mildly depend on the injection composition. In the middle panel of Fig. 2, we present the neutrino emission from the magnetar case $\left(P_{i}=0.6 \mathrm{~ms}, B=10^{15} \mathrm{G}\right)$, but with an injection composed of pure iron $(\mathrm{Fe})$ nuclei. Comparing it with the top panel one can tell that these two cases resulted in similar emission levels and spectrum shapes. Indeed, in a hardonuclear interaction with the center of momentum energy as high as $\sim \mathrm{TeV}$ or above, an Fe nucleus with energy $E_{\mathrm{Fe}}=56 E_{\mathrm{P}}$ (see Eqn. 2.3) is roughly equivalent to 56 protons each with energy $56 / 56 E_{\mathrm{P}}$ when producing pions. On the other hand, as $\dot{N}_{\mathrm{GJ}} \propto 1 / Z$, the injected numbers of Fe nuclei would be 26 times less than that of the protons. As a consequence, an injection of nucleus with a mass number $A$ and charge $Z$ would result in $\sim A / Z \sim 2$ times of neutrino flux, but $\sim A / A=1$ times of $E_{\nu, \max }$, compared to a pure $\mathrm{P}$ injection.

In the bottom panel of Fig. 2, we show the neutrino spectrum from a Crab-like pulsar with $B=10^{13} \mathrm{G}$ and $P_{i}=20 \mathrm{~ms}$ at $10 \mathrm{kpc}$. In this Crab case $\tau_{\mathrm{EM}}=1.2 \times 10^{10} B_{13}^{-2} P_{-1.7}^{2} \mathrm{~s} \gg$ $\tau_{\text {thin }}$, neutrinos are produced by a very small fraction of cosmic rays that were accelerated before the ejecta became optically thin. We thus adjusted the observation time to $10^{6}-10^{8}$ s. For $t \ll \tau_{\mathrm{EM}}, E_{\mathrm{CR}}=E(0)=4.2 \times 10^{15} A \kappa_{4}^{-1} \eta_{-0.5} B_{13} P_{-1.7}^{-2} \mathrm{eV}$ and the production rate of particles $\dot{N}_{\text {GJ }}$ is a constant. Thus, the neutrino emission in each time bin behaves similarly but with flux scaled to the size of the time window (as shown in the first three bins of Fig. 2 bottom). When time increases to $t>\tau_{\text {thin }}$ (the last two bins of Fig. 2 bottom), this feature dominates over the decreasing $f_{\pi}$. Compared to the previous case, pions and nuclei engaged in fewer interactions. With less boost from secondaries, the overall neutrino spectrum roughly follows $E^{-1.5}$.

Figure 3 present the light curves of neutrinos from the newborn pulsars. Note now the color codes have been changed to represent different neutrino energies. In the magnetar case (the top panel), neutrinos at different energies display similar shapes in light curves, though those with higher energies show up later in time. For each energy interval, the light curve peaks at the time when $f_{\text {sup }}=1$, specifically, $t_{\text {peak }, l c}=10^{4} E_{\nu, 13}^{1 / 3} P_{-3.2} \mathrm{~s}$. This is consistent 
with the simulation result, where $10 \mathrm{TeV}$ neutrinos peak around $10^{4} \mathrm{~s}$ and the peak time for neutrinos with higher energies increases by $E_{\nu}^{1 / 3}$. The decrement in the light curves at later times reflects the decrease of the average collision number a particle would encounter during its escape. In particular, neutrinos above $10^{17} \mathrm{eV}$ are produced after the environment gets optically thin, thus the flux is suppressed by $f_{\pi}<1$. In the crab case (the bottom panel), no neutrinos are produced above $\mathrm{PeV}$ due to the lack of cosmic ray acceleration. TeV neutrinos have less flux compared to those between $10 \mathrm{TeV}$ and PeV, being consistent with Fig. 2.

\section{Time-Dependent Neutrino Emissions}

If a nearby newborn pulsar can be resolved, its prompt neutrino emission would present a profile evolving with time. We study this emission profile in Section 5.1, and apply the scenario to young pulsars from recent supernova bursts in the local universe in Section 5.2.

\subsection{Flux level and Detectability}

Recall that at time $t$ after a pulsar birth at distance $\mathrm{D}$, neutrinos would emit at an intensity of

$$
E_{\nu}^{2} \Phi_{\nu}(t)=\frac{3}{8} E_{\mathrm{CR}}^{2} \frac{d N_{\mathrm{CR}}}{d E d t} \frac{1}{4 \pi D^{2}} f_{\pi}(t) f_{\mathrm{sup}}(t)
$$

Assume that we are looking at the source at the age of $t_{\mathrm{obs}}=1$ year, below we estimate this intensity again in the two cases of magnetar and crab. In the magnetar case, $t_{\mathrm{ob}} \gg \tau_{\mathrm{EM}}$, the typical neutrino energy reads $E_{\nu}=8.5 \times 10^{14} B_{15}^{-1} \eta_{-0.5} A \kappa_{4}^{-1} t_{\mathrm{yr}}^{-1} \mathrm{eV}$ and the flux would be

$$
\begin{aligned}
E_{\nu}^{2} \Phi_{\nu}= & 2.8 \times 10^{-9}\left(\frac{B}{10^{15} \mathrm{G}}\right)^{-2}\left(\frac{P}{0.6 \mathrm{~ms}}\right)^{2}\left(\frac{t_{\mathrm{obs}}}{1 \mathrm{yr}}\right)^{-4}\left(\frac{D}{10 \mathrm{kpc}}\right)^{-2} \\
& \times \eta_{-0.5} A \kappa_{4}^{-1} Z^{-1} M_{\mathrm{ej}, 1} \mathrm{TeV} \mathrm{cm}^{-2} \mathrm{~s}^{-1}
\end{aligned}
$$

In the crab case, $t_{\mathrm{ob}} \ll \tau_{\mathrm{EM}}$, so that $E_{\nu}\left(t_{\mathrm{obs}}\right) \approx E_{\nu}(0)=2.1 \times 10^{14} A \kappa_{4}^{-1} \eta_{-0.5} B_{13} P_{-1.7}^{-2} \mathrm{eV}$. The flux can be calculated as

$$
\begin{aligned}
E_{\nu}^{2} \Phi_{\nu}= & 4.4 \times 10^{-9}\left(\frac{P_{i}}{20 \mathrm{~ms}}\right)^{-4}\left(\frac{B}{10^{13} \mathrm{G}}\right)^{2}\left(\frac{t_{\mathrm{obs}}}{1 \mathrm{yr}}\right)^{-2}\left(\frac{D}{10 \mathrm{kpc}}\right)^{-2} \\
& \times \eta_{-0.5} A \kappa_{4}^{-1} Z^{-1} M_{\mathrm{ej}, 1} \mathrm{TeV} \mathrm{cm}^{-2} \mathrm{~s}^{-1}
\end{aligned}
$$

In the crab case, the pulsar would take a long time to spin down, therefore at a few years the neutrino flux only experiences the dilution of ejecta that decreases with $t^{-2}$; while in magnetar case the neutrino flux would be further impacted by the pulsar spin-down, and decreases with a faster rate of $t^{-4}$.

To demonstrate the flux evolution over time, we present in Figure 4 the neutrino flux level at 5 minutes, 1 day, 1 month, 1, 10 and 100 years after the birth of a pulsar, with $P_{i}, B$ scanning over the parameter space $\left(0.6 \mathrm{~ms} \leq \mathrm{P}_{\mathrm{i}} \leq 200 \mathrm{~ms}\right) \times\left(10^{12} \mathrm{G} \leq \mathrm{B} \leq 10^{16} \mathrm{G}\right)$. We fixed the source distance at $D=10 \mathrm{kpc}$. The red line in the last two cases (at 10 and 100 years) represents IceCube's median sensitivity at 90\% C.L., $\sim 10^{-12} \mathrm{TeV}^{-1} \mathrm{~cm}^{-2} \mathrm{~s}^{-1}$ for energies between $\mathrm{TeV}-\mathrm{PeV}$ in the northern sky based on 4 years of data [11]; this sensitivity is scaled to the corresponding observation time in the top four cases. At the very beginning, suppression from $\pi p$ interaction in the early environment is too fatal to allow the production of neutrinos. This suppression still distorts the flux at a few days and 

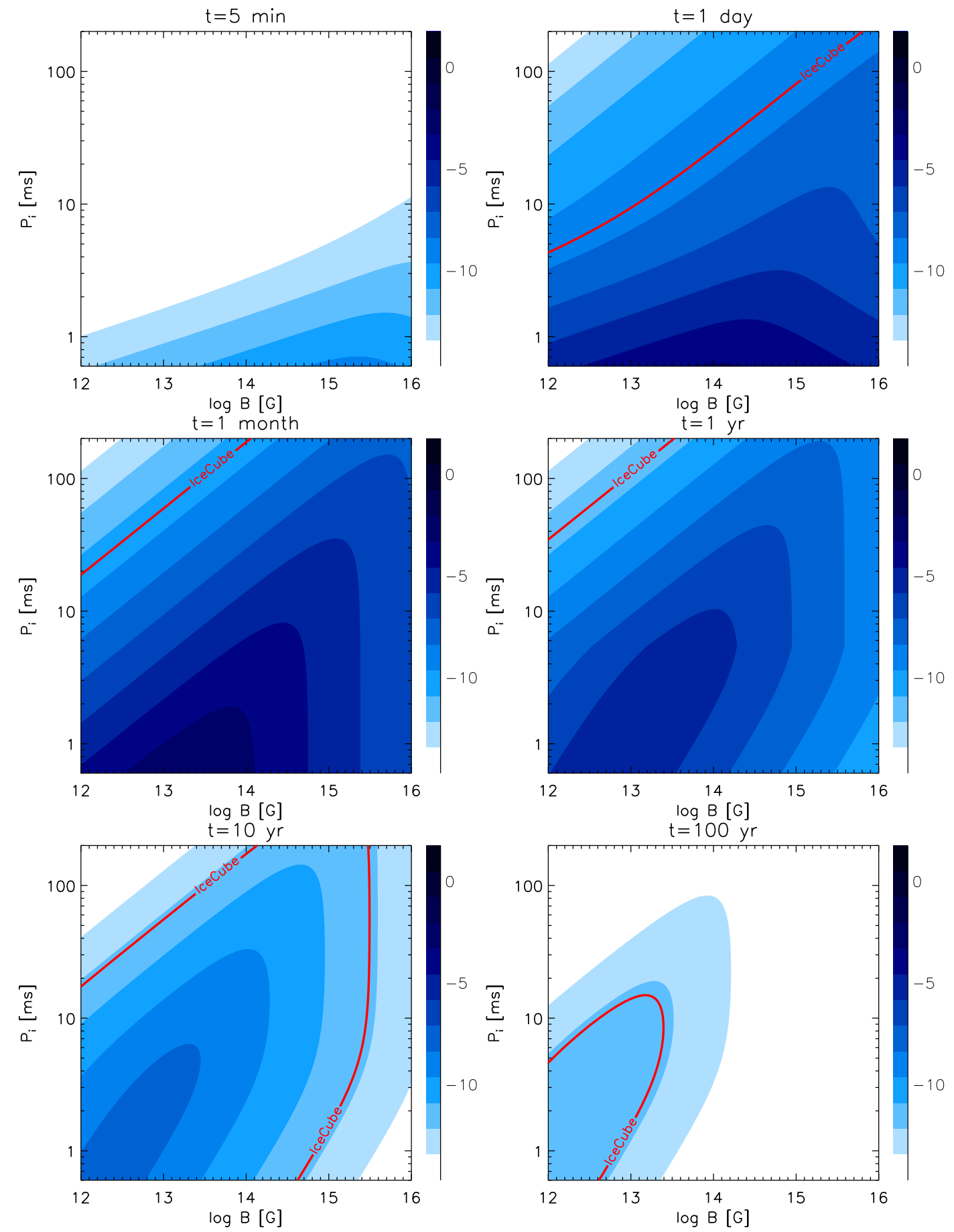

Figure 4. Neutrino flux $E_{\nu}^{2} \Phi_{\nu}$ at $\mathrm{t}=5$ minutes, 1 day, 1 month, 1,10 and 100 years after the birth of a pulsar with $P_{i}, B$ scanning over the parameter space $\left(0.6 \mathrm{~ms} \leq \mathrm{P}_{\mathrm{i}} \leq 200 \mathrm{~ms}\right) \times\left(10^{12} \mathrm{G} \leq\right.$ $\left.\mathrm{B} \leq 10^{16} \mathrm{G}\right)$. The flux is in unites of $\mathrm{TeV} \mathrm{s}^{-1} \mathrm{~cm}^{-2}$, shown in logarithmic scale with amplitude indicated in the color bar. The source distance is fixed at $10 \mathrm{kpc}$. Wind acceleration efficiency is set to be $\eta=0.3$. The red solid line indicates the medium point-source sensitivity of IceCube, $\left(E^{2} \Phi\right)_{\mathrm{IC}}=10^{-12} \mathrm{TeV} \mathrm{s}^{-1} \mathrm{~cm}^{-2}$ based on 4-year data [11] in the last two cases (at 10 and 100 years), but scaled to an observation time of 5 minutes, 1 day, 1 month, and 1 year in the top four cases. 
Table 2. Properties of Pulsars

\begin{tabular}{ccccccccc}
\hline \hline PSR & SNR & $\begin{array}{c}\text { Supernova } \\
\text { Type }\end{array}$ & $\begin{array}{c}\mathrm{D} \\
(\mathrm{kpc})\end{array}$ & $\begin{array}{c}P_{i} \\
(\mathrm{~ms})\end{array}$ & $\begin{array}{c}\mathrm{B} \\
\left(10^{12} \mathrm{G}\right)\end{array}$ & $\begin{array}{c}\text { Age } \\
(\mathrm{yr})\end{array}$ & $\begin{array}{c}E_{\exp } \\
\left(10^{51} \mathrm{erg}\right)\end{array}$ & $\begin{array}{c}M_{\mathrm{ej}} \\
\left(M_{\odot}\right)\end{array}$ \\
\hline B0531+21 & Crab & IIP & 2 & 20 & 4 & 950 & 1 & 9.5 \\
J0205+64 & 3C 58 & IIP & 3.2 & 50 & 4 & 2400 & 1 & 3.2 \\
J1846-03 & Kes 75 & Ib/c & 19 & 30 & 48 & 1000 & 2.1 & 16.4 \\
B1509-58 & MSH 15-52 & Ib/c & 5.2 & 10 & 14 & 1700 & 7 & 4 \\
\hline
\end{tabular}

finally becomes less important after around 1 month (see equation 2.9). At about 1 month, the flux reaches the maximum. As the ejecta was optically thick with $f_{\pi}=1$, the flux depended only on the neutrino energy, scaling as $E_{\nu}^{2} \propto \mu P_{i}^{-2}$. When $t \geq 1 \mathrm{yr}$ the effect of the decrease of $f_{\pi}$ added in. Recall that for pulsars with high angular velocity that satisfies $1 / 2 I \Omega_{i}^{2} \gg 10^{51} \mathrm{erg}$, ejecta expands with speed $\beta \propto \Omega_{i}$. Thus in the bottom half of the $t=1 \mathrm{yr}$ plot, $E_{\nu}^{2} \Phi_{\nu} \propto E_{\nu}^{2} f_{\mathrm{pp}} \propto \mu^{-1} P_{i}^{2}$. This shape maintained to $t=10 \mathrm{yr}$. Finally at $t=100 \mathrm{yr}$, the interaction probability decreased to $f_{\pi} \sim 10^{-4}$. In addition, due to the spin down the majority of pulsars failed to produce neutrinos above $1 \mathrm{TeV}$, to reach the optimized observation window of the IceCube Observatory [24].

\subsection{Existing nearby pulsars}

Four nearby young pulsar wind nebula and supernova remnants in which central pulsars have been identified are listed in Table 2. This list includes all the objects within $5 \mathrm{kpc}$ and with estimated initial spin period less than $50 \mathrm{~ms}$ in Table 1 of [25] except for Kes 75 . Kes 75 is shown because of its large magnetic field. These objects are known to actively interacting with ejecta $[17,25]$.

In Figure 5 we show the expected neutrino emission from these pulsars at their current ages. Among the list, neutrino flux from Crab pulsar has the highest flux, due to its young age and close distance. 3C58 has the lowest emission, as a result of the old age and the relatively slow spinning speed. For comparison, we also show the $5 \sigma$ differential discovery potentials of IceCube at declination $0^{\circ},-30^{\circ}$ and $+30^{\circ}$ [11]. The expected neutrino emissions from nearby young pulsars are far below the discovery sensitivities, consistent with the non-detection of point sources [11]. As a comparison, we show the neutrino flux from two future pulsars at their peak time, with yellow and red lines indicating magnetar and crab case respectively. These two cases would be easily observed by IceCube.

\section{Birth rate of detectable pulsars}

In this section we investigate the birth rate of pulsars that would be detectable by the IceCube Observatory. Assuming that a pulsar could at least be observed at the peak time $t_{\text {peak }}$ as defined in equation 2.9 , we define the maximum distance of a detectable pulsar by

$$
D_{\max }=\left(\left(E_{\nu}^{2} d N / d E d t\right)_{\text {peak }} / 4 \pi /\left(E_{\nu}^{2} \Phi_{\nu}\right)^{\operatorname{det}}\right)^{1 / 2}
$$

with $\left(E_{\nu}^{2} \Phi_{\nu}\right)^{\text {det }}$ the detector sensitivity.

The median IceCube sensitivity reads $\left(\Phi_{\nu}\right)^{\mathrm{IC}}=10^{-11} \mathrm{TeV}^{-1} \mathrm{~cm}^{-2} \mathrm{~s}^{-1}$ for energies above $100 \mathrm{TeV}$ and $\left(\Phi_{\nu}\right)^{\mathrm{IC}}=10^{-12} \mathrm{TeV}^{-1} \mathrm{~cm}^{-2} \mathrm{~s}^{-1}$ for energies between $1 \mathrm{TeV}-1 \mathrm{PeV}$ [11]. Taking 


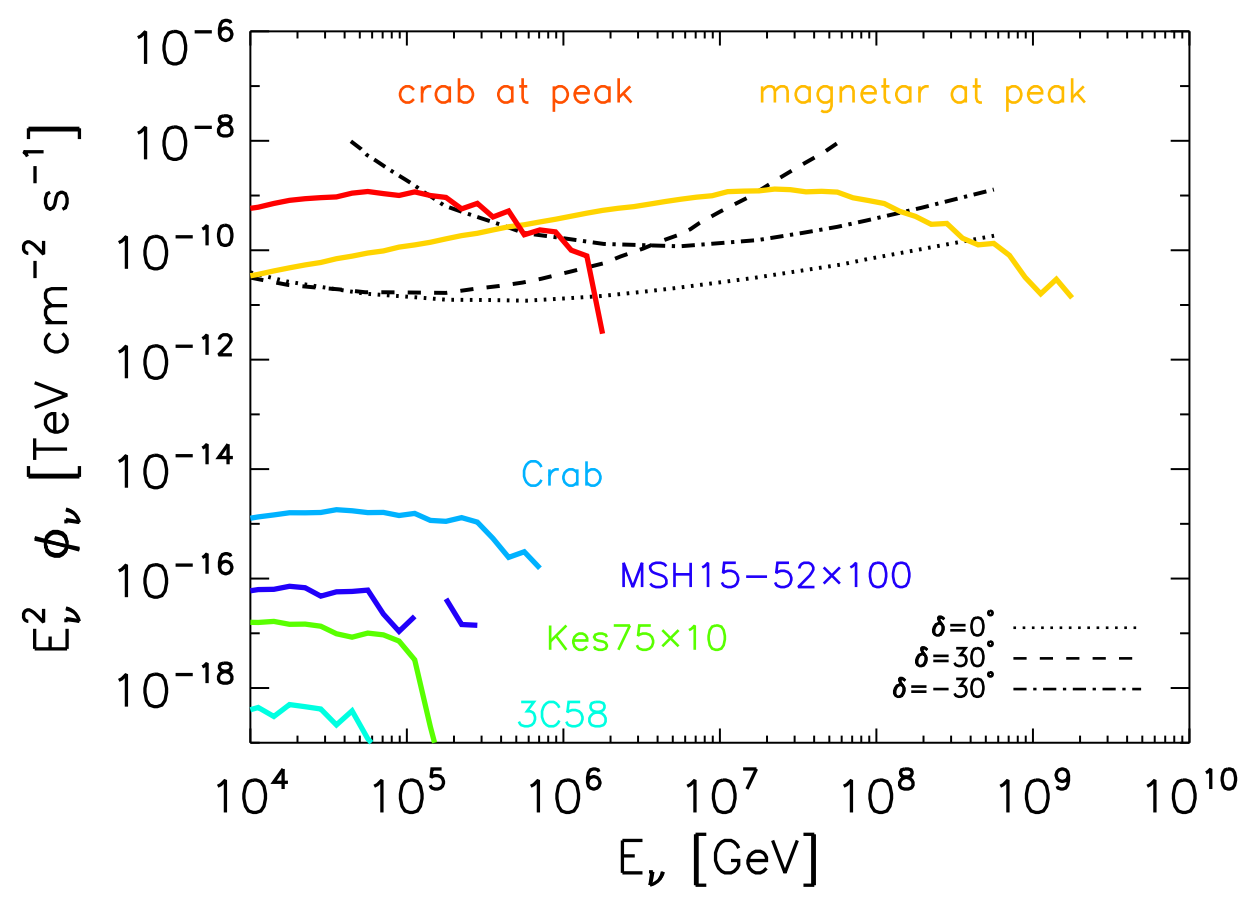

Figure 5. Expected neutrino spectra of four nearby young pulsars at their current ages, compared to the $5 \sigma$ differential discovery sensitivities of the IceCube Observatory [11]. As indicated in the legends, the pulsars include Crab pulsar, J0205, J1846 and B1509, with their properties summarized in Table 2. Note that the flux of MSH15-52 and Kes 75 was enlarged by 100 and 10 times accordingly to not overlap with other lines. Also plotted are the predicted emission from a fast-spinning magnetar located at $5 \mathrm{Mpc}$ at its neutrino peak time $t_{\text {peak }}=2.4 \times 10^{5} \mathrm{~s}$ and a Crab-like pulsar at $10 \mathrm{kpc}$ at $t_{\text {peak }}=2.1 \times 10^{5} \mathrm{~s}$. Their input parameters are listed in Table 1 .

this sensitivity, $D_{\max }$ can be estimated to be, in the magnetar case,

$$
D_{\max }=87.2 B_{15}^{-3 / 4} \eta_{-0.5}^{1 / 4} A^{1 / 4} \kappa_{4}^{-1 / 4} Z^{-1 / 2} M_{\mathrm{ej}, 1}^{-1 / 4} P_{i,-3.2}^{-3 / 4}\left(\frac{\Phi_{\nu}^{\text {det }}}{\Phi_{\nu}^{\text {IC }}}\right)^{1 / 2} \mathrm{Mpc}
$$

and in the crab case,

$$
D_{\max }=0.5 B_{13} P_{-1.7}^{-2} \eta_{-0.5}^{1 / 2} A^{1 / 2} \kappa_{4}^{-1 / 2} Z^{-1 / 2}\left(\frac{\Phi_{\nu}^{\mathrm{det}}}{\Phi_{\nu}^{\mathrm{IC}}}\right)^{1 / 2} \mathrm{Mpc}
$$

According to [26], the initial spin periods and magnetic fields of newborn isolated neutron stars displace a distribution $f\left(P_{i}, B\right)$, with $P_{i}$ normally distributed, centered at $\left\langle P_{i}\right\rangle=300 \mathrm{~ms}$ with standard derivation $\sigma_{P_{i}}=150 \mathrm{~ms}$; B log-normally distributed with $\langle\log B\rangle=12.65$ and $\sigma_{\log B}=0.55$. A lower limit of the initial spin period, $P_{i, \min } \sim 0.6-1 \mathrm{~ms}$ is required for a stable configuration of a neutron star $[23,27]$. In this work, we set $P_{i, \min }$ to be $0.6 \mathrm{~ms}$.

Based on the pulsar distribution, below we investigate the birth rate of pulsars that could be detected by the IceCube Observatory. We first investigate the detection upper limit in Sec. 6.1, and then refine this upper limit by considering explicitly the contribution from pulsars in nearby clusters (Sec. 6.2) and pulsars in the Local Group (Sec. 6.3). 


\subsection{Upper limit}

The local core-collapse supernova has a formation rate of $\rho_{0}=10^{-4} \mathrm{Mpc}^{-3} \mathrm{yr}^{-1}$ [28]. Taken this rate, we can calculate the birth rate of the detectable pulsars in the universe by counting the formation rate of each pulsar in its own maximum volume and summing the population over the $(\mathrm{P}, \mathrm{B})$ probability space:

$$
\bar{\Re}_{\text {limit }}=\int d P_{i} \int d B \rho_{0} \frac{4 \pi}{3} D_{\max }\left(P_{i}, B\right)^{3} w\left(P_{i}, B\right) f\left(P_{i}, B\right)
$$

Here we only count the pulsars that could contribute to the IceCube sensitivity window by setting a window function $w\left(P_{i}, B\right)=\mathbb{1}\left(E_{\nu} \geq \mathrm{TeV}\right)$.

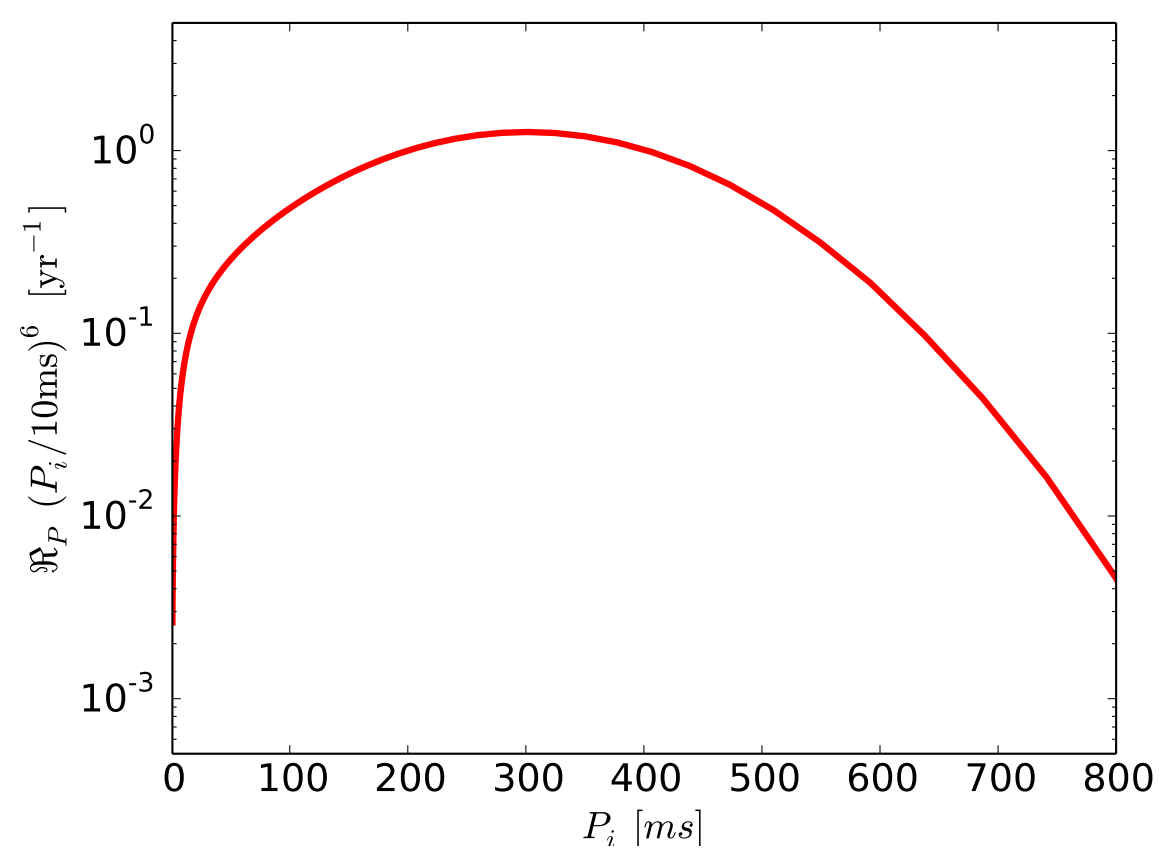

Figure 6. Dependence of the upper limit of birth rate of detectable pulsars on the initial spin periods $P_{i}$. $\Re_{P}$ is defined in Eqn. 6.5 which has marginalized over the magnetic field distribution. Note that the y-axis is scaled by $\left(P_{i} / 10 \mathrm{~ms}\right)^{6}$ for presentation purposes (see Sec. 6.1 for more details).

$\bar{\Re}_{\text {limit }}$ significantly depends on the minimum spin periods $P_{i, \min }$ and the acceleration efficiency $\eta$. To understand its dependence on $P_{i, \min }$, in Fig. 6 we show $\Re_{P}$ as a function of $P_{i}$, with $\Re_{P}$ denoting the inner integral of Eqn. 6.4 which marginalizes over the magnetic field distribution:

$$
\Re_{P}\left(P_{i}\right)=\int d B \rho_{0} \frac{4 \pi}{3} D_{\max }\left(P_{i}, B\right)^{3} w\left(P_{i}, B\right) f\left(P_{i}, B\right)
$$

With the y-axis of Fig. 6 scaled by $\left(P_{i} / 10 \mathrm{~ms}\right)^{6}$, one could easily find that $\Re_{P} P_{i}^{6}$ roughly agrees with the $P_{i}$ distribution, which is a Gaussian distribution with a mean of $300 \mathrm{~ms}$ and a standard derivation of $150 \mathrm{~ms}$. The reason is that as the magnetic field distribution strongly peaks at $10^{12-13} \mathrm{G}, \Re_{P}$ is mostly contributed by Crab-like pulsars which have $D_{\text {max }} \propto P^{-2}$, thus $\Re_{P} \propto D_{\text {max }}^{3} f\left(P_{i}\right) \propto f\left(P_{i}\right) P_{i}^{-6} . \Re_{P} P_{i}^{6}$ cuts off sharply at low $P_{i}$ further due to the 
contribution of fast-spinning magnetars, which have a particularly large $D_{\max }$ but scaling to $P_{i}^{-3 / 4}$. Fig. 6 indicates that the birth rate of observable extragalactic pulsars is heavily dominated by the fast-spinning pulsars, and thus strongly depends on $P_{i, \min }$. Moreover, the upper limit depends on the wind acceleration efficiency by $\bar{\Re}_{\text {limit }} \sim \eta^{1.3}$ due to the weighted contribution from magnetars and crabs. Results of $\bar{\Re}_{\text {limit }}$ with different inputs of $P_{i, \text { min }}$ and $\eta$ are listed in Table 3. Considering $P_{i \text {, min }}=0.6 \mathrm{~ms}$ and $\eta=0.3$, we find $\bar{\Re}_{\text {limit }}=0.29 \mathrm{yr}^{-1}$.

Table 3. Upper Limits on the Birth Rate of Detectable Pulsars in year ${ }^{-1}$

\begin{tabular}{|c|c|c|c|}
\hline$P_{i, \min } \longrightarrow$ & 0.1 & 0.3 & 0.7 \\
\hline $0.6 \mathrm{~ms}$ & 0.07 & 0.29 & 0.82 \\
\hline $1 \mathrm{~ms}$ & 0.01 & 0.04 & 0.12 \\
\hline
\end{tabular}

Notice that $\bar{\Re}_{\text {limit }}$ is an optimized upper limit because: i) although $t_{\text {peak }}$ depends on $P_{i}$ and $B$ and varies from months to tens of years, pulsars have been assumed be always detectable at their brightest point; ii) [18] found a normalization factor $f_{s} \approx 5 \%$ if using UHECR measurements to confine the pulsar population, which means $\sim 5 \%$ of the pulsar population is required to have the right configuration to successfully inject and accelerate ions. If taking this confinement, the upper limit would drop to $5 \%$ of what calculated from Eqn. 6.4. iii) $\bar{\Re}_{\text {limit }}$ has ideally assumed a continuous pulsar distribution up to $\sim 100 \mathrm{Mpc}$ in the local universe, while in reality the local large-scale structure is not uniform. We will further demonstrate this point next.

\subsection{Pulsars in nearby clusters}

To take into account that the local large-scale structure is not uniform, we refine our estimation of the extragalactic pulsar detection rate by considering explicitly the contribution from nearby clusters. For a nearby cluster at $D_{\text {cluster }}$ with a total pulsar birth rate of $\rho_{\text {cluster }}$, the rate of pulsars that can be observed from such a distance can be estimated by

$$
\bar{\Re}_{\text {cluster }}=\rho_{\text {cluster }} \int w\left(P_{i}, B\right) P_{\text {obs }}^{\text {cluster }}\left(P_{i}, B\right) f\left(P_{i}, B\right) d P_{i} d B
$$

$P_{\text {obs }}^{\text {cluster }}$ is the observational chance of a pulsar inside the cluster. Since a pulsar can be observable only when its maximum distance exceeds the distance of the cluster, we have

$$
P_{\text {obs }}^{\text {cluster }}= \begin{cases}1 & \text { if } D_{\max } \geq D_{\text {cluster }} \\ 0 & \text { if } D_{\max }<D_{\text {cluster }}\end{cases}
$$

Take the closest Virgo Cluster at $D_{\text {Virgo }} \sim 16.5 \mathrm{Mpc}$ for example. Within its total newborn pulsar population which has a rate of $\sim 20 \mathrm{yr}^{-1}[29], 0.16 \%$ have $D_{\max } \geq D_{\text {Virgo }}$ and thus can be seen from the Earth. This corresponds to a birth rate of $0.032 \mathrm{yr}^{-1}$. Note that we have assumed $P_{i, \min }=0.6 \mathrm{~ms}, \eta=0.3$, and that the sources are from the central region of the Virgo Cluster.

With the same calculation, we find that the other nearby clusters - Centaurus Cluster at $52 \mathrm{Mpc}$, Perseus Cluster at $74 \mathrm{Mpc}$, and Coma Cluster at $102 \mathrm{Mpc}$ have 0.058\%, 0.039\%, and $0.025 \%$ of their local pulsars observable. Along with the Virgo Cluster, and assuming each of them has a local core-collapse SN rate of $20 \mathrm{yr}^{-1}$, we get a total detection rate of 0.06 per year from pulsars in the nearby clusters. 


\subsection{Pulsars in the Local Group}

Observations suggest that a total of 4.5 core-collapse supernova are expected per century in the Local group, including 2.5 from our Galaxy $\left(\rho_{\text {Gal }}=0.025 \mathrm{yr}^{-1}\right), 0.5$ from the Magellanic Clouds, and 1.5 from M31 plus M33 [26, 30, 31].

We first consider the birth rate of observable Galactic newborn pulsars. A pulsar would definitely be observed if it has $D_{\max }$ greater than the size of our Galaxy, $R_{\text {Gal }} \approx 15 \mathrm{kpc}$. On the other hand, if a pulsar has $D_{\max }<R_{\mathrm{Gal}}$, it still has a probability of $\left(D_{\max } / R_{\mathrm{Gal}}\right)^{3}$ to be observed, with a very rough assumption that the occurrence of core-collapse supernova is uniform inside the Galaxy. The above two cases summarize to an observation probability $P_{\text {obs }}^{\mathrm{Gal}}\left(P_{i}, B\right) \equiv \max \left(\left(D_{\max } / R_{\mathrm{Gal}}\right)^{3}, 1\right)$, which yields a total birth rate of detectable pulsars in the Galaxy to be

$$
\begin{aligned}
\bar{\Re}_{\mathrm{Gal}} & =\rho_{\mathrm{Gal}} \int w\left(P_{i}, B\right) P_{\mathrm{obs}}^{\mathrm{Gal}}\left(P_{i}, B\right) f\left(P_{i}, B\right) d P_{i} d B \\
& =0.01 \mathrm{yr}^{-1}
\end{aligned}
$$

assuming that $P_{i, \min }=0.6 \mathrm{~ms}$ and $\eta=0.3$. The same calculation can be applied to the birth rate of pulsars in the Local Group, which has a size of $R_{\mathrm{LC}} \approx 1 \mathrm{Mpc}$ [32]. However, due to the larger distance, nearby galaxies only enhance $\bar{\Re}_{\text {Gal }}$ by $4.8 \times 10^{-4} \mathrm{yr}^{-1}$.

\section{Conclusions and Discussion}

In this work we have investigated high energy neutrino emissions from individual newborn pulsars in the Local Universe. Through both theoretical and numerical methods, we examined the hadronuclear interactions between cosmic rays and the source ambience left over from the supernova explosion and reported on the detectability and emission profiles of the high-energy neutrino products. We found that

I) unlike the $E^{-1}$ spectrum of the injection cosmic rays, the energy spectrum of the neutrinos has a softer shape, following $\sim E^{-2}$ for a fast-spinning magnetar, and $\sim E^{-1.5}$ for a Crab-like pulsar. The modifications to the spectrum mainly originate from the high-order interactions of secondary ions and charged pion products in the early environment;

II) The flux level and shape of time-integrated neutrino spectrum are not significantly sensitive to the chemical composition of the injected cosmic rays;

III) Applying the scenario to known nearby pulsars found their neutrino detectability to be below the sensitivities of the current generation of detectors and consistent with the negative results of point source searches;

IV) The birth rate of pulsars with neutrino emissions detectable by the IceCube Observatory is constrained with an upper limit of 0.29 per year. In particular, the birth rate of detectable Galactic pulsars is estimated to be 0.01 per year, and that of pulsars from nearby clusters is found to be 0.06 per year.

One caveat of our work is that we did not take into account the radiation fields of the pulsar. A relativistic Fermi process is known to occur in the termination shock of the pulsar wind nebula $[33,34]$. Nonthermal radiations from electron and positions accelerated in the 
termination shocks are expected to lead to photodisintegration of the cosmic rays, in addition to the hadronuclear channel discussed in this work. Figure 4 of [14] compared the neutrino emission from $p \gamma$ and $p p$ interactions to show that for a population of pulsars with identical parameters, the neutrino production from $p \gamma$ interactions is 2-3 orders less than that from $p p$ interactions, suggesting that the $p \gamma$ channel is sub-dominant to the $p p$ channel in neutrino productions. Moreover, in extremely radiative magnetars, a fraction of the outflow energy may dissipate as thermal radiation via the shocks [35-38]. The photomeson production rate with these thermal photons is $\tau_{p \gamma, \text { th }}=12 E_{52}^{3 / 4} \eta_{\gamma,-1}^{3 / 4} t_{\mathrm{yr}}^{-5 / 4} \beta_{-1.5}^{-5 / 4}$, where $\eta_{\gamma} \sim 0.1$ is the fraction of total pulsar energy that goes into thermal photons [13]. Notice that $\tau_{p \gamma \text {,th }}$ decreases slower than $\tau_{\mathrm{Np}}$ with $\tau_{p \gamma, \text { th }} \propto t^{-5 / 4}$, so the thermal photons could produce neutrinos comparable to those with hadronic origins after about a year. However, thermal photons are not as important in less radiative pulsars like Crab.

When considering the cosmic ray interaction with the ejecta, we ignored the delay of cosmic rays in the magnetic field of pulsar wind nebula. The larmor radius of particles reads $r_{L} \approx 10^{11}(E / 1 \mathrm{PeV}) \mathrm{Z}^{-1}\left(\mathrm{~B}_{\mathrm{pwn}} / 30 \mathrm{G}\right)^{-1} \mathrm{~cm}$, in a nebula with field strength $B_{\mathrm{pwn}} \sim$ $37 P_{-3}^{-5 / 2} B_{13}^{3}\left(t / \tau_{\mathrm{EM}}\right)^{-7 / 4} \mathrm{G}[14]$. At small $\mathrm{t}$, the size of the supernova ejecta is not significantly greater than $r_{L}$, then particles escape recti-linearly with negligible time delay. As time increases $R_{\mathrm{ej}} \gg r_{L}$, particles diffuse in the structure and exit with a deflection of order unity, $\delta \theta \approx\left(1+2 r_{L}^{2} / R \lambda\right)^{-1} \sim 1^{\circ}$, and a time delay comparable to the crossing time, $\delta t=$ $R \delta \theta^{2} / 6 c \sim R / c$ [39]. This adds to the pion production rate $f_{\pi}$ a factor of $\sim 2$. However, this little increase in $f_{\pi}$ would be dominated by the fast decrease of the number density of target baryons in the ejecta. Therefore the effect from the magnetic field of the pulsar wind nebula is considerably small.

One aim of the present work is to provide an observational template for neutrino emissions from future pulsar sources in the local universe. Compared to other transient sources like Gamma-ray Bursts, fast-spinning pulsars display unique features in high-energy neutrino emissions, including time-variable spectrum shapes, insensitivity to the chemical composition of injected parent particles, and an $\sim E^{-1.5}$ spectrum index for the majority of the population. Such neutrinos might be detected by current and future generations of high-energy neutrino observatories like IceCube [11], KM3NeT [40], and by future ultrahigh energy neutrino detectors like JEM-EUSO [41], Askaryan Radio Array (ARA) [42], and the Antarctic Ross Ice-Shelf ANtenna Neutrino Array (ARIANNA) [43], thereby providing a unique way for understanding particle acceleration in a newborn pulsar.

\section{Acknowledgments}

The author would like to thank the anonymous referee for helpful comments and suggestions. The author acknowledges S. Dodelson, K. Kotera, N. Neilson, A. Olinto, P. Privitera and S. Wakely for very fruitful discussions. This work was supported by the NSF grant PHY-1068696 at the University of Chicago, and the Kavli Institute for Cosmological Physics through grant NSF PHY-1125897 and an endowment from the Kavli Foundation. KF acknowledge financial support from NASA 11-APRA-0066 at the University of Chicago. This work made use of computing resources and support provided by the Research Computing Center at the University of Chicago. 


\section{References}

[1] J. P. Ostriker and J. E. Gunn, On the Nature of Pulsars. I. Theory, Astrophys.J. 157 (Sept., 1969) 1395.

[2] M. Hoshino, J. Arons, Y. A. Gallant, and A. B. Langdon, Relativistic magnetosonic shock waves in synchrotron sources - Shock structure and nonthermal acceleration of positrons, ApJ 390 (May, 1992) 454-479.

[3] Y. A. Gallant and J. Arons, Structure of relativistic shocks in pulsar winds: A model of the wisps in the Crab Nebula, ApJ 435 (Nov., 1994) 230-260.

[4] J. E. Gunn and J. P. Ostriker, Acceleration of high-energy cosmic rays by pulsars, Phys. Rev. Lett. 22 (Apr, 1969) 728-731.

[5] P. Blasi, R. I. Epstein, and A. V. Olinto, Ultra-High-Energy Cosmic Rays from Young Neutron Star Winds, ApJ Letters 533 (Apr., 2000) L123-L126.

[6] J. Arons, Magnetars in the Metagalaxy: An Origin for Ultra-High-Energy Cosmic Rays in the Nearby Universe, ApJ 589 (June, 2003) 871-892.

[7] K. Fang, K. Kotera, and A. V. Olinto, Newly Born Pulsars as Sources of Ultrahigh Energy Cosmic Rays, The Astrophysical Journal 750 (May, 2012) 118, [arXiv:1201.5197].

[8] IceCube Collaboration, M. Aartsen et al., Evidence for High-Energy Extraterrestrial Neutrinos at the IceCube Detector, Science 342 (2013), no. 6161 1242856, [arXiv:1311.5238].

[9] IceCube Collaboration, M. Aartsen et al., Observation of High-Energy Astrophysical Neutrinos in Three Years of IceCube Data, arXiv:1405.5303.

[10] M. G. Aartsen et al., Search for time-independent neutrino emission from astrophysical sources with 3 yr of icecube data, The Astrophysical Journal 779 (2013), no. 2132.

[11] IceCube Collaboration, M. Aartsen et al., Searches for Extended and Point-like Neutrino Sources with Four Years of IceCube Data, arXiv:1406.6757.

[12] K. Fang, K. Kotera, K. Murase, and A. V. Olinto, A decisive test for the young pulsar origin of ultrahigh energy cosmic rays with IceCube, arXiv:1311.2044.

[13] K. Murase, P. Mészáros, and B. Zhang, Probing the birth of fast rotating magnetars through high-energy neutrinos, Phys. Rev. D 79 (May, 2009) 103001-+, [arXiv:0904.2509].

[14] M. Lemoine, K. Kotera, and J. Petri, Ultra-high energy cosmic ray acceleration at the termination shock of pulsar winds, JCAP (in prep.).

[15] P. Goldreich and W. H. Julian, Pulsar Electrodynamics, ApJ 157 (Aug., 1969) 869.

[16] J. A. Hibschman and J. Arons, Pair Production Multiplicities in Rotation-powered Pulsars, ApJ 560 (Oct., 2001) 871-884, [astro-ph/0107209].

[17] N. Bucciantini, J. Arons, and E. Amato, Modeling the spectral evolution of PWNe inside SNRs, ArXiv e-prints (May, 2010) [arXiv:1005.1831].

[18] K. Fang, K. Kotera, and A. V. Olinto, Ultrahigh energy cosmic ray nuclei from extragalactic pulsars and the effect of their Galactic counterparts, JCAP 3 (Mar., 2013) 10, [arXiv: 1302.4482].

[19] K. Kotera, Ultrahigh energy cosmic ray acceleration in newly born magnetars and their associated gravitational wave signatures, Phys.Rev.D 84 (July, 2011) 023002, [arXiv:1106.3060].

[20] S. Razzaque, P. Mészáros, and E. Waxman, Neutrino tomography of gamma ray bursts and massive stellar collapses, Phys. Rev. D 68 (Oct, 2003) 083001. 
[21] K. Werner, F.-M. Liu, and T. Pierog, Parton ladder splitting and the rapidity dependence of transverse momentum spectra in deuteron-gold collisions at the bnl relativistic heavy ion collider, Phys. Rev. C $\mathbf{7 4}$ (Oct, 2006) 044902.

[22] T. Bergmann, R. Engel, D. Heck, N. Kalmykov, S. Ostapchenko, T. Pierog, T. Thouw, and K. Werner, One-dimensional hybrid approach to extensive air shower simulation, Astroparticle Physics 26 (2007), no. 6420 - 432.

[23] P. Haensel, J. P. Lasota, and J. L. Zdunik, On the minimum period of uniformly rotating neutron stars, Astron. Astrophys. 344 (Apr., 1999) 151-153.

[24] IceCube Collaboration, A. Achterberg et al., First Year Performance of The IceCube Neutrino Telescope, Astropart.Phys. 26 (2006) 155-173, [astro-ph/0604450].

[25] R. A. Chevalier, Young Core-Collapse Supernova Remnants and Their Supernovae, ApJ 619 (Feb., 2005) 839-855, [astro-ph/0409013].

[26] C.-A. Faucher-Giguère and V. M. Kaspi, Birth and Evolution of Isolated Radio Pulsars, ApJ 643 (May, 2006) 332-355.

[27] J. W. T. Hessels, S. M. Ransom, I. H. Stairs, P. C. C. Freire, V. M. Kaspi, and F. Camilo, A Radio Pulsar Spinning at 716 Hz, Science 311 (Mar., 2006) 1901-1904, [astro-ph/0601337].

[28] R. G. Izzard, E. Ramirez-Ruiz, and C. A. Tout, Formation rates of core collapse SNe and GRBs, Mon.Not.Roy.Astron.Soc. 348 (2004) 1215, [astro-ph/0311463].

[29] L. Stella, S. Dall'Osso, G. L. Israel, and A. Vecchio, Gravitational Radiation from Newborn Magnetars in the Virgo Cluster, ApJL 634 (Dec., 2005) L165-L168, [astro-ph/0511068].

[30] G. A. Tammann, W. Loeffler, and A. Schroeder, The Galactic supernova rate, ApJS 92 (June, 1994) 487-493.

[31] S. M. Adams, C. S. Kochanek, J. F. Beacom, M. R. Vagins, and K. Z. Stanek, Observing the Next Galactic Supernova, ApJ 778 (Dec., 2013) 164, [arXiv:1306.0559].

[32] I. Karachentsev and O. Kashibadze, Masses of the local group and of the m81 group estimated from distortions in the local velocity field, Astrophysics 49 (2006), no. 1 3-18.

[33] A. M. Atoyan and F. A. Aharonian, On the mechanisms of gamma radiation in the Crab Nebula, Mon.Not.Roy.Astron.Soc. 278 (Jan., 1996) 525-541.

[34] J. G. Kirk, Y. Lyubarsky, and J. Petri, The theory of pulsar winds and nebulae, astro-ph/0703116.

[35] T. A. Thompson, P. Chang, and E. Quataert, Magnetar spindown, hyper-energetic supernovae, and gamma ray bursts, Astrophys.J. 611 (2004) 380-393, [astro-ph/0401555].

[36] B. D. Metzger, T. A. Thompson, and E. Quataert, Proto-Neutron Star Winds with Magnetic Fields and Rotation, Astrophys.J. 659 (2007) 561-579, [astro-ph/0608682].

[37] N. Bucciantini, T. A. Thompson, J. Arons, E. Quataert, and L. Del Zanna, Relativistic mhd winds from rotating neutron stars, Mon.Not.Roy.Astron.Soc. 368 (2006) 1717-1734, [astro-ph/0602475].

[38] D. Kasen and L. Bildsten, Supernova Light Curves Powered by Young Magnetars, Astrophy.J. 717 (July, 2010) 245-249, [arXiv:0911.0680].

[39] K. Kotera and M. Lemoine, The optical depth of the Universe for ultra-high energy cosmic ray scattering in the magnetized large scale structure, Phys.Rev. D77 (2008) 123003, [arXiv:0801.1450].

[40] KM3NeT, http://www.km3net.org/home.php, .

[41] The JEM-EUSO Collaboration, The JEM-EUSO Mission: Status and Prospects in 2011, arXiv: 1204.5065. 
[42] ARA Collaboration, Design and initial performance of the Askaryan Radio Array prototype EeV neutrino detector at the South Pole, Astroparticle Physics 35 (Feb., 2012) 457-477, [arXiv: 1105.2854].

[43] S. W. Barwick, ARIANNA: A New Concept for UHE Neutrino Detection, J.Phys.Conf.Ser. 60 (2007) 276-283, [astro-ph/0610631]. 